\title{
Low-intensity ultrasound enhances the antitumor effects of doxorubicin on hepatocellular carcinoma cells through the ROS-miR-21-PTEN axis
}

\author{
CHUNHUA XIA ${ }^{1,2}$, HUABEI ZENG ${ }^{1}$ and YANFEN ZHENG ${ }^{2}$ \\ ${ }^{1}$ Department of Ultrasound, Suqian Obstetrics and Gynecology Hospital, Suqian, Jiangsu 223800; ${ }^{2}$ Department of Ultrasound, \\ School of Imaging of Baotou Medical College, Inner Mongolia University of Science and Technology, \\ Baotou, Inner Mongolia 014060, P.R. China
}

Received June 21, 2018; Accepted March 6, 2019

DOI: $10.3892 / \mathrm{mmr} .2020 .10936$

\begin{abstract}
Hepatocellular carcinoma (HCC) is a type of liver cancer and is a leading cause of cancer-associated mortality. In China, 466,000 patients are diagnosed with HCC and it is responsible for $\sim 422,000$ cases of mortality each year. Surgery is the most effective treatment available; however it is only suitable for patients with early-stage HCC. Chemotherapy has been confirmed as a necessary treatment for patients with advanced HCC, although drug resistance may limit its clinical outcome. Low intensity ultrasound (LIUS) represents a novel therapeutic approach to treat patients with HCC; however, its underlying molecular mechanism remains unclear. In the present study, cell viability, apoptosis and reactive oxygen species (ROS) generation were determined via Cell Counting Kit-8, flow cytometry and 2',7'-dichlorofluorescein diacetate assays, respectively. The expression of miRNA in HCC cells following exposure to LIUS and doxorubicin (Dox) was analyzed using a microarray and reverse transcription-quantitative polymerase chain reaction analysis. It was revealed treatment with LIUS in combination with Dox was able to induce apoptosis of Huh7 cells, increasing the intracellular levels of reactive oxygen species (ROS) and malondialdehyde. Glutathione peroxidase and superoxide dismutase 1 are ROS-scavenging enzymes, which serve important roles in the oxidative balance, preventing oxidative stress. The protein expression levels of these two enzymes were significantly decreased following treatment with LIUS combined with Dox. The present results suggested that LIUS may decrease Dox resistance in HCC cells and that LIUS may be combined
\end{abstract}

Correspondence to: Professor Huabei Zeng, Department of Ultrasound, Suqian Obstetrics and Gynecology Hospital, 88 Pingan Road, Suqian, Jiangsu 223800, P.R. China

E-mail: huabeizenghb@163.com

Key words: low-intensity ultrasound, reactive oxygen species, microRNA-21, phosphatase and tensin homolog, chemotherapy, resistance with chemotherapy to treat HCC. By performing microarray analysis, the expression levels of microRNA-21 (miR-21) were decreased following treatment with LIUS combined with Dox. Functional experiments showed that knockdown of miR-21 enhanced the antitumor activity of Dox, whereas overexpression of miR-21 reversed these effects. Phosphatase and tensin homolog (PTEN), a well-known tumor suppressor, was revealed to be a direct target of miR-21, and its translation was suppressed by miR-21. Finally, it was determined that combined treatment of LIUS and Dox induced anticancer effects by blocking the activation of the AKT/mTOR pathway, as demonstrated by the downregulation of phosphorylated (p-)AKT and p-mTOR; N-acetylcysteine, a general ROS inhibitor reversed the suppressive effects on the AKT/mTOR pathway mediated by LIUS and Dox. Collectively, the present results suggested that LIUS increased cell sensitivity to Dox via the ROS/miR-21/PTEN pathway. Chemotherapy combined with LIUS may represent a novel effective therapeutic strategy to treat patients with advanced $\mathrm{HCC}$.

\section{Introduction}

Hepatocellular carcinoma (HCC) is a highly prevalent and lethal disease, which poses a threat to human health. HCC is the fifth most common malignancy worldwide and the 5-year survival rate of patients with HCC is $<20 \%$ (1). However, the genetic factors and pathogenesis of HCC remain unclear, and liver resection is the only available curative treatment (2). Notably, surgical treatment only benefits patients diagnosed with early-stage $\mathrm{HCC}$, and liver resection is not effective in patients with late-stage $\operatorname{HCC}(2,3)$.

Chemotherapy is an effective strategy to increase the survival rate of patients with late-stage HCC (4). Notably, targeted therapies for HCC have been approved for clinical use (4). Sorafenib and doxorubicin (Dox) are widely used chemical drugs that represent standard therapies for patients with advanced HCC (5). However, drug resistance mechanisms may limit the effectiveness of chemotherapy in patients with HCC (6). Therefore, the identification of novel clinical strategies able to promote chemotherapeutic sensitivity is required. 
Dox is a type of anthracycline, which inhibits protein translation by interacting with DNA and RNA (7). Dox is the most common chemotherapy drug for the treatment of various types of cancer, including breast cancer, gastric carcinoma, liver cancer, lung cancer and lymphoma (8). However, the molecular mechanism underlying Dox function remains unclear. Notably, chemoresistance to Dox represents a major challenge for the treatment of HCC. A previous study demonstrated that AMP-activated protein kinase family member 5 (ARK5) is able to modulate the resistance of HCC to Dox via epithelial-mesenchymal transition (EMT) (9). A previous study identified that semaphorins regulate cell migration, enhancing the resistance of HCC to Dox (10). Additionally, salinomycin, an ionophore antibiotic, reverses the resistance of HCC to Dox by inhibiting the $\beta$-catenin/TCF complex and activating forkhead box $\mathrm{O} 3$ (11). Collectively, these previous studies suggested that chemoresistance in HCC may be a multifactorial mechanism that requires further investigation.

A previous study demonstrated that low intensity ultrasound (LIUS) enhances the anticancer effects of chemotherapy (12). LIUS can treat solid tumors via sonodynamic therapy, ultrasound-mediated chemotherapy, ultrasound-mediated gene delivery and antivascular ultrasound therapy (12). A previous study demonstrated that LIUS, in combination with chemical compounds, suppresses proliferation of tongue squamous carcinoma cells (13). In addition, it has been demonstrated that LIUS increases Dox uptake, and inhibits cancer cell proliferation and migration (14). Although various studies have observed an association between treatment with LIUS and tumor suppression, the mechanism underlying the antitumor effects of LIUS remains unclear. Therefore, understanding the molecular mechanism underlying LIUS may facilitate the development of clinical strategies combining chemotherapy with LIUS to treat cancer.

Reactive oxygen species (ROS) are involved in numerous pathophysiological processes. A previous study demonstrated that ROS, by modulating the expression levels of certain microRNAs (miRNAs/miRs), may regulate gene expression in tumor cells (15). Oxidative stress has been reported to induce the expression of miRNAs belonging to the miR-200 family, and the crosstalk between ROS signaling and miR-200 increases oxidative stress-mediated liver cell death (16). Notably, the ROS-MYC proto-oncogene, bHLH transcription factor-miR-27 pathway increases HCC cell proliferation and liver cancer progression (17). These findings indicate that LIUS may affect the expression of miRNAs via the production of ROS. The present study hypothesized that dysregulated miRNA expression induced by ROS accumulation may represent the mechanism underlying enhanced Dox sensitivity following treatment with LIUS.

In the present study, a novel regulatory pathway consisting of LIUS, ROS and miRNAs was identified in HCC cells. The present results suggested that LIUS was able to significantly increase sensitivity to Dox by activating the ROS pathway. Furthermore, ROS decreased the expression levels of miR-21, resulting in increased expression levels of PTEN and HCC cell apoptosis. Therefore, the present results suggested that LIUS together with Dox may represent a novel strategy to decrease chemoresistance in HCC, improving the effectiveness of chemotherapy in clinical settings.

\section{Materials and methods}

Cells and ultrasound device. Huh7 cells were purchased from The Shanghai Institute of Cell Biology, Chinese Academy of Sciences (Shanghai, China) and cultured in Dulbecco's modified Eagle's medium (DMEM; Gibco; Thermo Fisher Scientific, Inc., Waltham, MA, USA) supplemented with $10 \%$ fetal bovine serum (FBS; Gibco; Thermo Fisher Scientific, Inc.) and $1 \%$ penicillin-streptomycin (Gibco; Thermo Fisher Scientific, Inc.) in an incubator with a humidified atmosphere and $5 \% \mathrm{CO}_{2}$ at $37^{\circ} \mathrm{C}$. The different concentrations $(0-1.5 \mu \mathrm{g} / \mathrm{ml})$ of Dox (Wako Pure Chemical Industries, Ltd; Osaka, Japan) was added into cells, and cells were treated for $24 \mathrm{~h}$, as previously described (18). Huh7 cells ( $1 \times 10^{4}$ cells) were cultured in 3.5-cm diameter dishes (Corning, Inc., Corning, NY, USA) and placed on an ultrasonic transducer (Onda Corporation, Sunnyvale, CA, USA). LIUS waves of varying intensities (diameter: $40 \mathrm{~mm}$; center frequency: $1.1 \mathrm{MHz}$; duty factor: 20\%; repetition frequency: $100 \mathrm{~Hz}$ ) were transmitted for $15 \mathrm{~min}$ through the bottom of the cultured dishes via a $2.5-\mathrm{cm}$ thick aluminum block in a humidified $37^{\circ} \mathrm{C}$ incubator with $5 \% \mathrm{CO}_{2}$. Untreated cells served as controls. In certain experiments, the ROS scavenger $\mathrm{N}$-acetylcysteine (NAC; $10 \mathrm{mM}$, Sigma-Aldrich; Merck KGaA, Darmstadt, Germany) was added to cells $1 \mathrm{~h}$ prior to the administration of Dox at $37^{\circ} \mathrm{C}$; after $24 \mathrm{~h}$ of incubation, cell suspensions were immediately subjected to LIUS exposure. After the treatment, the cells were collected for further analyses.

Cell viability assay. Cells were treated as aforementioned. Cells were seeded at 5,000 cells/well in 96-well plates. After $24 \mathrm{~h}$, the medium was replaced with DMEM supplemented with $10 \%$ FBS and Dox $(0,0.1,0.2,0.4,0.8$ or $1.5 \mu \mathrm{g} / \mathrm{ml})$. Cells were cultured in an incubator for $24 \mathrm{~h}$ at $37^{\circ} \mathrm{C}$ with $5 \% \mathrm{CO}_{2}$. Cell viability was measured using a Cell Counting Kit- 8 assay (Dojindo Molecular Technologies, Inc., Kumamoto, Japan), according to the manufacturer's protocol. A microplate reader (MRX II; Dynex Technologies, Inc., Chantilly, VA, USA) was used to measure the optical density at $450 \mathrm{~nm}$.

Malondialdehyde (MDA) measurement. The level of lipid peroxidation was assessed by measuring MDA levels using the thiobarbituric acid reactive substance (TBARS) according to the method of Zhang et al (19). In brief, Huh7 cells were treated with LIUS and/or Dox for $24 \mathrm{~h}$, and then the cells were homogenized on ice in lysis buffer (cat. no. P0013B; Beyotime Institute of Biotechnology, Haimen, China) and then centrifuged at $13,000 \mathrm{x} \mathrm{g}$ for $10 \mathrm{~min}$ at $4^{\circ} \mathrm{C}$ to remove insoluble material. Supernatant $(200 \mu \mathrm{l})$ were placed into a micro-centrifuge tube and $600 \mu \mathrm{l}$ of the TBARS solution then added. This mixture was incubated at $95^{\circ} \mathrm{C}$ for $60 \mathrm{~min}$ and cooled to room temperature in an ice bath for $10 \mathrm{~min}$. Finally, $200 \mu \mathrm{l}$ was pipetted into each well of a 96-well plate, and the absorbance at $532 \mathrm{~nm}$ was measured using a spectrophotometer (UV-1800 UV-vis spectrophotometer, SHIMADZU Corporation, Tokyo, Japan). A standard curve was prepared using various concentrations of 1,1,3,3-tetraethoxypropane (1-10 nM). TBARS levels were indicated in nM. TBA was procured from Sigma-Aldrich (Merck KGaA). Other chemicals required, such as EDTA and trichloroacetic acid were procured from Merck KGaA. 
Cell apoptosis assay. Cell apoptosis was assessed by staining the cells with the BD Pharmingen ${ }^{\mathrm{TM}}$ Annexin V-fluorescein isothiocyanate and propidium iodide kit (BD Biosciences), according to the manufacturer's protocol. The cells were analyzed with a FACSCalibur flow cytometer (BD Biosciences) and then analyzed by FlowJo 8.7.1 software (FlowJo LLC). Staining cells simultaneously with Annexin V-FITC (green fluorescence) and the non-vital dye PI (red fluorescence) allowed the discrimination of viable cells (FITC-PI'), early apoptotic $\left(\mathrm{FITC}^{+} \mathrm{PI}^{-}\right)$, and late apoptotic or necrotic cells $\left(\mathrm{FITC}^{+} \mathrm{PI}^{+}\right)$. Finally, the apoptotic rate was calculated from the percentage of early + late apoptotic cells.

ROS detection. The generation of ROS was assessed using 2',7'-DCFH diacetate (DCFH-DA; Sigma-Aldrich; Merck $\mathrm{KGaA}$ ). Briefly, at the end of treatment, the cell culture medium was discarded and the cells were incubated with DCFH $(20 \mu \mathrm{mol} / \mathrm{l})$ for $30 \mathrm{~min}$ at $37^{\circ} \mathrm{C}$, followed by two washes with PBS. Then the DCFH-DA stain detecting ROS production was observed using a fluorescence microscope (magnification, x200; Nikon Corporation). Fluorescence was read at $485 \mathrm{~nm}$ for excitation and $530 \mathrm{~nm}$ for emission with an Infinite M200 Microplate Reader (Tecan Group, Ltd.) and analyzed with BD FACSDiva (version 6.2; BD Biosciences) software.

Microarrayanalysis. Total RNA was extracted from Huh7 cells using TRIzol ${ }^{\circledR}$ reagent (Invitrogen; Thermo Fisher Scientific, Inc.), according to the manufacturer's protocol. Briefly, the quantity of RNA samples was evaluated via NanoDrop ${ }^{\mathrm{TM}}$ ND-1000 spectrophotometry (NanoDrop Technologies; Thermo Fisher Scientific, Inc.). Total RNA (200 ng) was labeled with fluorescence dye hy3 or hy5 using a miRCURY Hy3/Hy5 Power Labeling kit (cat. no. 208031-A) and hybridized on the miRCURY ${ }^{\mathrm{TM}}$ LNA Array (v.18.0), both obtained from Exiqon (Qiagen, Inc.) according to the manufacturer's protocol. Data were analyzed using GeneSpring software version 7.3 (Agilent Technologies, Inc.). The miRNAs with intensities $\geq 50$ were used to calculate a normalization factor in all samples. Normalization was performed using median normalization. The miRNA expression profiles (heatmaps) were determined using MEV software (version 4.6; http:// mev.tm4.org/\#/welcome).

Reverse transcription-quantitative polymerase chain reaction $(R T-q P C R)$. miRNA was prepared using the miRNeasy Mini kit (Qiagen, Inc.) and total RNA was prepared using TRIzol reagent (Thermo Fisher Scientific, Inc.) according to the manufacturer's protocol. For miRNA reverse transcription, cDNA was synthesized using TaqMan ${ }^{\circledR}$ miRNA reverse transcription kit (Applied Biosystems; Thermo Fisher Scientific, Inc.) at $42^{\circ} \mathrm{C}$ for $1 \mathrm{~h}$. For mRNA reverse transcription, cDNA was synthesized using the Oligo dT primer (Takara Biotechnology Co., Ltd.) at $42^{\circ} \mathrm{C}$ for $1 \mathrm{~h}$. qPCR was performed using an SYBR Green PCR mix (Takara Biotechnology Co., Ltd.). qPCR was conducted as follows: $95^{\circ} \mathrm{C}$ for $15 \mathrm{~min}$, followed by 40 cycles of $94^{\circ} \mathrm{C}$ for $15 \mathrm{sec}, 55^{\circ} \mathrm{C}$ for $30 \mathrm{sec}$ and $70^{\circ} \mathrm{C}$ for $30 \mathrm{sec}$, and a final extension step at $72^{\circ} \mathrm{C}$ for $5 \mathrm{~min}$. The following primers were used for RT-qPCR analysis: miR-21 forward (F), 5'-GCC CGCTAGCTTATCAGACTGATG-3' and miR-21 reverse (R), 5'-CAGTGCAGGGTCCGAGGT-3'; U6 F, 5'-TGCGGGTGC
TCGCTTCGCAGC-3' and U6 R, 5'-CCAGTGCAGGGTCCG AGGT-3'; PTEN F, 5'-TTGGCGGTGTCATAATGTCT-3' and PTEN R, 5'-GCAGAAAGACTTGAAGGCGTA-3'; GAPDH F, 5'-AGGTCGGTGTGAACGGATTTG-3' and GAPDH R, 5'-TGTAGACCATGTAGTTGAGGTCA-3' The RT-qPCR assays were performed in triplicate and the relative expression levels were calculated based on the $2^{-\Delta \Delta \mathrm{Cq}}$ method (20).

Transfection. When Huh7 cells in 6-well plates had grown to $\sim 80 \%$ confluence, miR-21 mimics $(20 \mathrm{nM})$ or miR-21 inhibitor $(20 \mathrm{nM})$ were transfected into cells at $37^{\circ} \mathrm{C}$ for $48 \mathrm{~h}$, using Lipofectamine ${ }^{\circledR} 2000$ (Invitrogen; Thermo Fisher Scientific, Inc.). After $4 \mathrm{~h}$, the transfection medium was discarded. Cells were washed with serum-free DMEM, then cultured in DMEM supplemented with $10 \%$ FBS. miR-21 mimics, mimics negative control (NC), miR-21 inhibitor and inhibitor NC were obtained from Guangzhou RiBoBio Co., Ltd. The sequences were as follows: miR-21 inhibitor, 5'-AUCGAAUAGUCU GACUACAACU-3'; miR-21 mimics, 5'-UAGCUUAUCAGA CUGAUGUUGA-3'; mimics NC, 5'-CCCCCCCCCCCCCCC CCCCC-3'; inhibitor NC, 5'-CAGUACUUUUGUAGUACA A-3'. Cells were harvested after $24 \mathrm{~h}$ for further analyses.

Western blotting. Huh7 cells were lysed in lysis buffer (Tris $50 \mathrm{mM}, \mathrm{pH}$ 7.4, $\mathrm{NaCl} 150 \mathrm{mM}, 1 \%$ Triton X-100 and EDTA $1 \mathrm{mM}, \mathrm{pH}$ 8.0) containing cOmplete ${ }^{\mathrm{TM}}$ Mini Protease Inhibitor (Roche Diagnostics) for $20 \mathrm{~min}$ on ice, and cell debris was removed by centrifugation at $15,000 \times \mathrm{g}$ for $20 \mathrm{~min}$ at $4{ }^{\circ} \mathrm{C}$. The protein concentration was determined using a bicinchoninic acid assay kit (Beyotime Institute of Biotechnology). The proteins (30 $\mu \mathrm{g} /$ lane) were separated via $10 \%$ SDS-PAGE and transferred onto PVDF membranes (EMD Millipore). Membranes were blocked with $5 \%$ milk for $2 \mathrm{~h}$ at room temperature, and then the membranes were incubated overnight at $4^{\circ} \mathrm{C}$ with primary antibodies against superoxide dismutase 1 (1:1,000; SOD-1; cat. no. sc-101523; Santa Cruz Biotechnology, Inc., Dallas, TX, USA), glutathione peroxidase (1:1,000; GPx; cat. no. sc-133160; Santa Cruz Biotechnology, Inc.), PTEN (1:1,000; cat. no. 9188; Cell Signaling Technology, Inc., Danvers, MA, USA), phosphorylated (p-)AKT (1:1,000; cat. no. 4060; Cell Signaling Technology, Inc.), AKT (1:1,000; cat. no. 4685; Cell Signaling Technology, Inc.), p-mTOR (1:1,000; cat. no. 5536; Cell Signaling Technology, Inc.), mTOR (1:1,000; cat. no. 2983; Cell Signaling Technology, Inc.) and $\beta$-actin (1:1,000; cat. no. 3700; Cell Signaling Technology, Inc.), followed by incubation with horseradish peroxidase-conjugated goat anti-rabbit or mouse IgG secondary antibodies (1:10,000; cat. nos. ab205718 or ab6789; Abcam, Cambridge, UK) at room temperature for $2 \mathrm{~h}$. The protein bands were detected using an enhanced chemiluminescence kit (Pierce; Thermo Fisher Scientific, Inc.). Semi-quantification was performed using ImageJ version 1.46 (National Institutes of Health, Bethesda, MD, USA).

Bioinformatics. Online miRNA prediction websites were used for initial analyses, including TargetScan 7.0 (http://www. targetscan.org/) and miRanda (http://www.microrna.org/).

Luciferase assay. The predicted and mutated sequences targeting the 3'-untranslated region (UTR) of PTEN were 
A
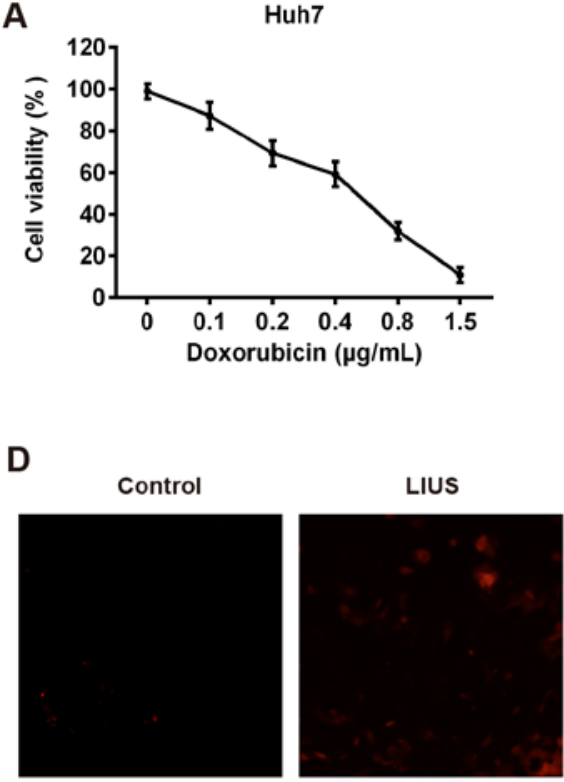

E

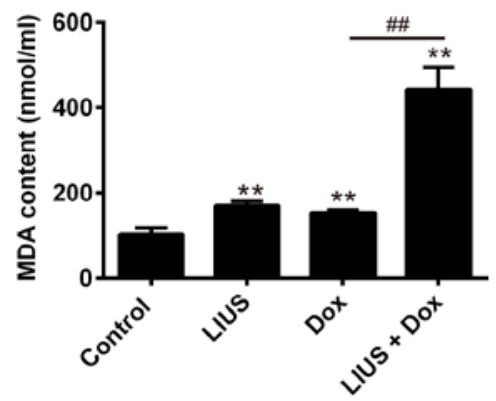

B

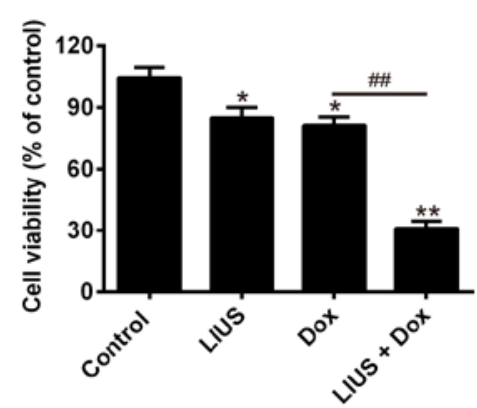

Dox

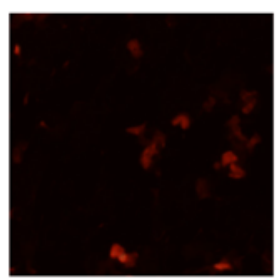

$\mathbf{F}$

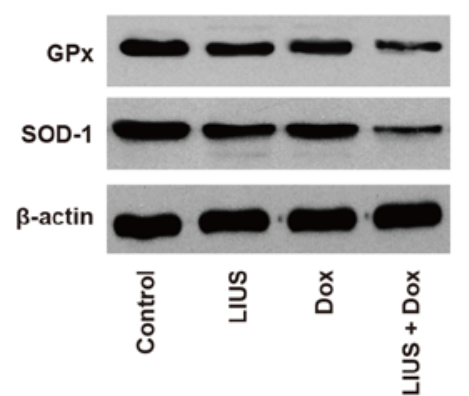

C
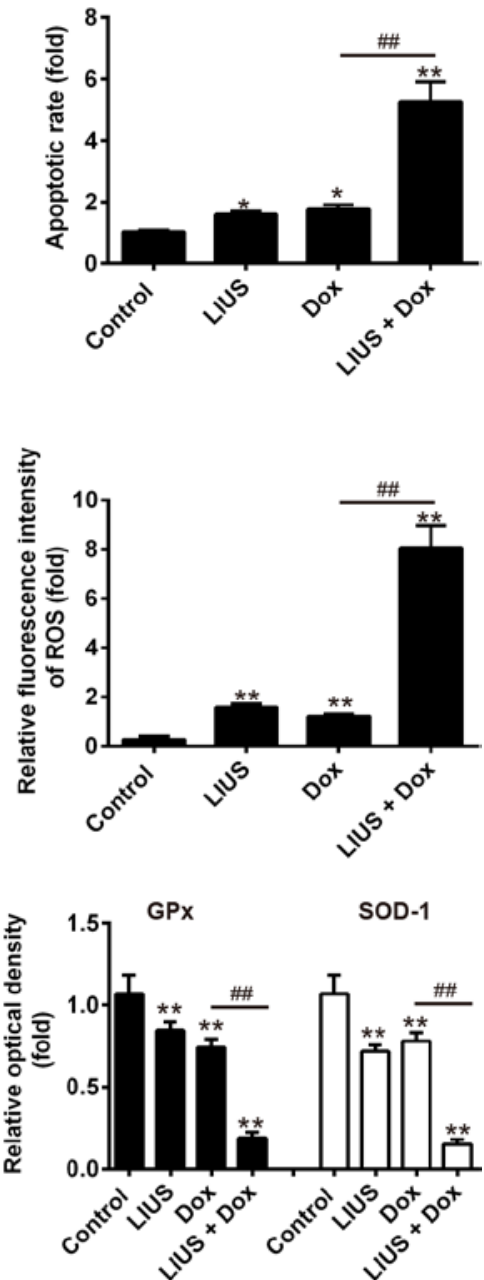

Figure 1. Dox combined with LIUS promotes apoptosis of hepatocellular carcinoma cells. (A) Cell viability assay in Huh7 cells following treatment with increasing concentrations of Dox (0-1.5 $\mu \mathrm{g} / \mathrm{ml})$. (B) Viability of Huh7 cells following treatment with LIUS and/or Dox for $24 \mathrm{~h}$. (C) Apoptosis of Huh7 cells was detected by propidium iodide/Annexin V staining and flow cytometry following treatment with LIUS and/or Dox. (D) Intracellular ROS levels (red fluorescence) were assessed by MitoSOX staining in Huh7 cells following treatment with LIUS and/or Dox. Magnification, x200. (E) MDA levels were examined following treatment with LIUS and/or Dox. (F) Protein expression levels of SOD-1 and GPx were assessed by western blotting. "P<0.05, ** $<0.01$ vs. control group; ${ }^{\#} \mathrm{P}<0.01$ vs. Dox group. Dox, doxorubicin; GPx, glutathione peroxidase; LIUS, low intensity ultrasound; MDA, malondialdehyde; ROS, reactive oxygen species; SOD-1, superoxide dismutase 1.

amplified and cloned into the pGL3 vector (Promega Corporation). pGL3-PTEN-3'-UTR wild-type (WT) and pGL3-PTEN-3'-UTR mutated (Mut) were synthesized by GenePharma. Huh7 cells $\left(1-2 \times 10^{5}\right.$ cells per well) were co-transfected with $10 \mathrm{ng}$ pGL3 luciferase vectors and $20 \mathrm{ng}$ Renilla vector (pRL-TK; Promega Corporation), together with $20 \mathrm{nM}$ miR-21 inhibitor, $20 \mathrm{nM}$ miR-21 mimics, $20 \mathrm{nM}$ mimics NC or $20 \mathrm{nM}$ inhibitor NC using Lipofectamine ${ }^{\circledR} 2000$ (Invitrogen; Thermo Fisher Scientific, Inc.) for $24 \mathrm{~h}$ at $37^{\circ} \mathrm{C}$. Luciferase activity was detected using the Dual-Luciferase Reporter Assay system (Promega Corporation). Firefly luciferase activity was normalized to Renilla luciferase activity.

Statistical analysis. Data are presented as the means \pm standard deviation. Experiments were performed at least three times in triplicate. Differences were analyzed with one-way analysis of variance among multiple groups followed by Tukey's post hoc test. $\mathrm{P}<0.05$ was considered to indicate a statistically significant difference.

\section{Results}

Dox combined with LIUS promotes apoptosis of HCC cells. Huh7 is a HCC cell line that is sensitive to Dox. To examine the effectiveness of Dox in suppressing tumor growth, Huh7 cells were treated with various doses of Dox and cell viability was measured after $24 \mathrm{~h}$. The present results demonstrated that treatment with Dox decreased the survival rate of Huh7 cells in a dose-dependent manner (Fig. 1A). The half maximal inhibitory concentration of Dox was $0.57 \mu \mathrm{g} / \mathrm{ml}$; in contrast, only minor reductions in viability were observed following treatment with $0.1 \mu \mathrm{g} / \mathrm{ml}$ Dox. Therefore, $0.1 \mu \mathrm{g} / \mathrm{ml}$ Dox was selected as a working concentration to investigate the ability of LIUS to promote sensitivity to Dox. As presented in Fig. 1B and C, compared with Dox treatment alone, cell 
A

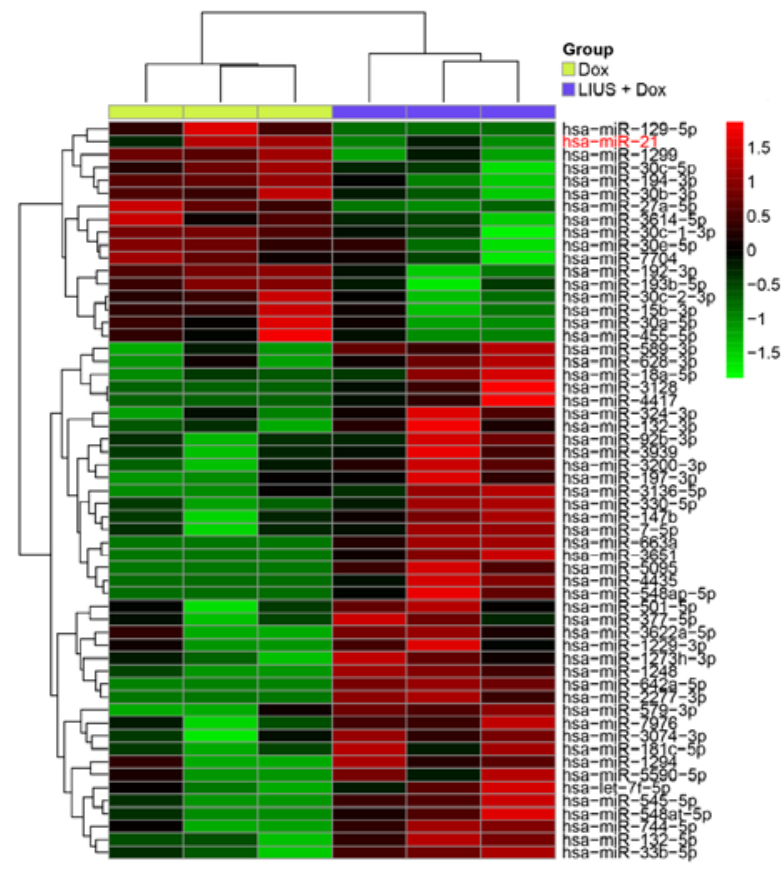

B
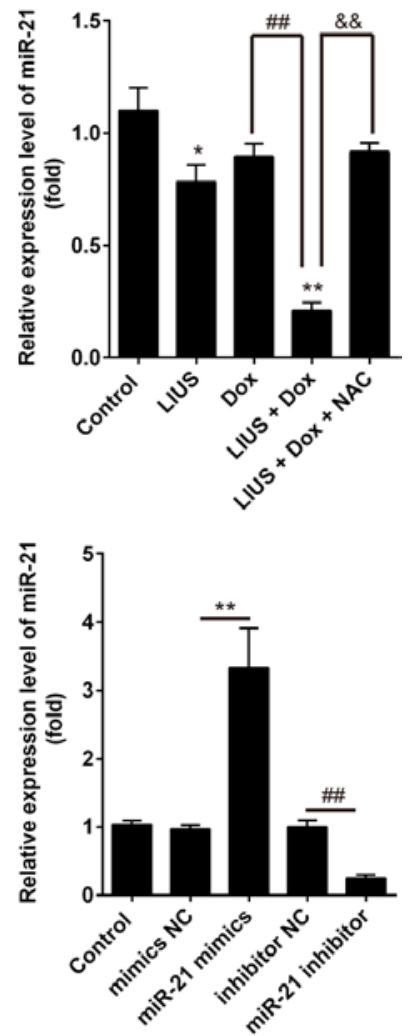

Figure 2. Dox combined with LIUS downregulates miR-21 expression in hepatocellular carcinoma cells via the accumulation of reactive oxygen species. (A) Heatmap of normalized expression levels of miRNAs in Huh7 cells treated with Dox and/or LIUS. (B) Expression levels of miR-21 following treatment with various compounds for $24 \mathrm{~h}$, as assessed by RT-qPCR. ${ }^{*} \mathrm{P}<0.05$ vs. control group; ${ }^{\# \#} \mathrm{P}<0.01$ vs. Dox group; \&\&P<0.01 vs. LIUS + Dox group. (C) Transfection efficiency of miR-21 mimics, miR-21 inhibitor and respective controls, as assessed by RT-qPCR. ${ }^{* *} \mathrm{P}<0.01$ vs. mimics NC; ${ }^{\# \#} \mathrm{P}<0.01$ vs. inhibitor NC. Dox, doxorubicin; LIUS, low intensity ultrasound; miR, microRNA; NAC, N-acetylcysteine; NC, negative control; RT-qPCR, reverse transcription-quantitative polymerase chain reaction.

viability was significantly reduced and the rate of apoptosis was significantly increased in the LIUS + Dox group, consistent with a previous study (14). The present results suggested that LIUS may enhance HCC cell apoptosis in combination with chemotherapy. A previous study reported that treatment with LIUS increases intracellular ROS accumulation (21). Subsequently, the association between ROS and LIUS-induced apoptosis was investigated. ROS in Huh7 cells were detected by MitoSOX staining. The present study revealed that treatment with LIUS or Dox alone could increase the intracellular levels of ROS. Notably, ROS accumulation was significantly enhanced following combined treatment with LIUS and Dox (Fig. 1D). MDA is a marker of oxidative stress, and its intracellular levels were slightly increased in response to LIUS or DOX treatment. However, following combined treatment with LIUS and Dox, the concentration of MDA exhibited a 2-fold increase compared with single treatments (Fig. 1E). GPx and SOD-1 are ROS-scavenging enzymes that serve important roles in the oxidant/antioxidant balance, and are thus able to prevent oxidative stress. To further investigate the antioxidative response, the protein expression levels of GPx and SOD-1 were assessed by western blotting. The protein expression levels of GPx and SOD-1 were significantly decreased following combined treatment with LIUS and Dox (Fig. 1F). The present results suggested that LIUS combined with Dox increased apoptosis and ROS accumulation in Huh7 cells.
Dox combined with LIUS downregulates the expression levels of miR-21 in HCC cells via ROS. To investigate the mechanisms underlying LIUS-induced apoptosis, the miRNA expression profile of Huh7 cells following treatment with Dox alone or in combination with LIUS was investigated by microarray analysis. The microarray results suggested that LIUS combined with Dox affected the expression levels of certain miRNAs compared with Dox treatment alone (Fig. 2A). Among the miRNAs downregulated following combined treatment with LIUS and Dox, the expression levels of miR-21 were markedly decreased. A previous study demonstrated that miR-21 may serve as an oncogene with a role in cancer pathogenesis, invasion and metastasis (22). Additionally, miR-21 has been identified to mediate chemotherapy resistance in HCC cells (23), and to increase HCC cell growth and invasion (24). A previous study suggested that miR-21 may be used as a biomarker associated with poor prognosis in patients with HCC (25). Therefore, miR-21 was selected for further experiments. RT-qPCR was performed to validate the expression levels of miR-21 in Huh7 cells following single and combined treatments. In line with the microarray results, the expression levels of miR-21 were significantly decreased following combined treatment with LIUS and Dox compared with single treatments (Fig. 2B). Notably, NAC, a ROS inhibitor, restored the expression levels of miR-21 in Huh7 cells. The present results suggested that LIUS decreased the expression 
A
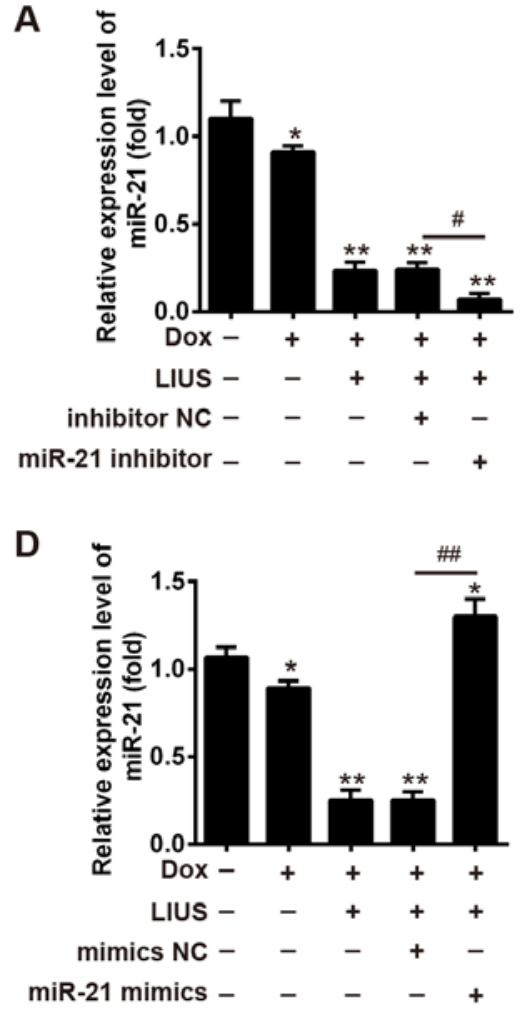

B

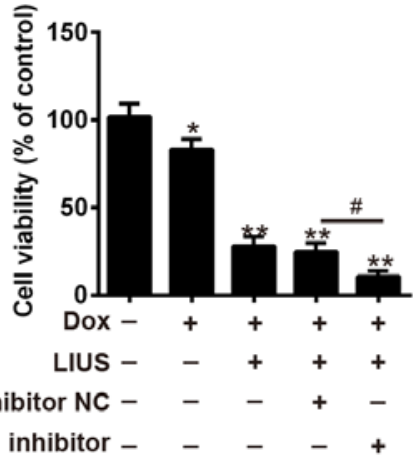

E

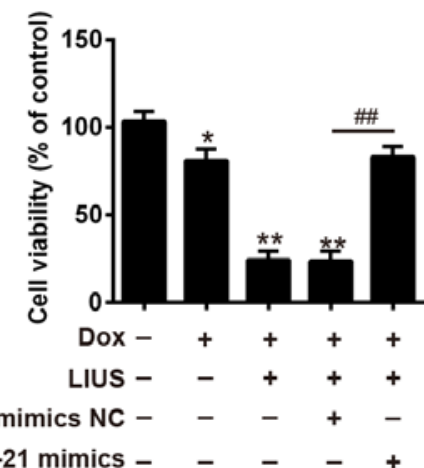

C

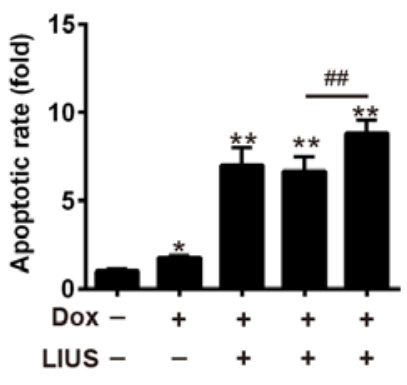

inhibitor NC - --+miR-21 inhibitor $-\quad+\quad-\quad+\quad+$

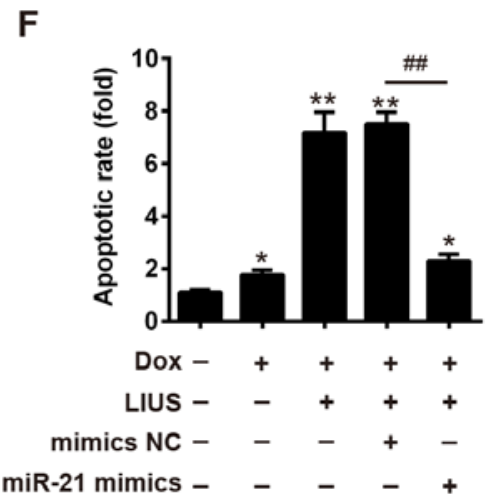

Figure 3. miR-21 regulates apoptosis following combined treatment with LIUS and Dox. (A and D) miR-21 expression was detected in Dox + LIUS-treated cells transfected with miR-21 inhibitor or mimics by reverse transcription-quantitative polymerase chain reaction. (B and E) Viability of Dox + LIUS-treated Huh7 cells transfected with miR-21 inhibitor or mimics was assessed by Cell Counting kit-8. (C and F) Apoptosis of Dox + LIUS-treated Huh7 cells transfected with miR-21 inhibitor or mimics was assessed by flow cytometry. ${ }^{*} \mathrm{P}<0.05,{ }^{* *} \mathrm{P}<0.05$ vs. control group; ${ }^{*} \mathrm{P}<0.05,{ }^{\# \#} \mathrm{P}<0.01$ vs. Dox + LIUS + mimics/inhibitor NC group. Dox, doxorubicin; LIUS, low intensity ultrasound; miR-21, microRNA-21; NC, negative control.

levels of miR-21 in Dox-treated cells via activation of the ROS pathway. In order to investigate the role of miR-21 in the effect of Dox and LIUS on cell survival, miR-21 was overexpressed or silenced using mimics or inhibitor, respectively. Post-transfection with miR-21 mimics or miR-21 inhibitor, the expression levels of miR-21 were significantly increased or decreased, respectively (Fig. 2C).

miR-21 regulates the effects of Dox and LIUS on HCC cell apoptosis. The present study hypothesized that Dox combined with LIUS may affect HCC cell survival via the ROS/miR-21 pathway. To examine the function of miR-21 on cell viability and apoptosis following treatment with Dox and/or LIUS, miR-21 inhibitor or miR-21 mimics were transfected into Huh7 cells, and cell viability and apoptosis were investigated (Fig. 3). In Huh7 cells cotreated with LIUS and Dox, the expression levels of miR-21 were decreased and increased following transfection with miR-21 inhibitor and mimics, respectively (Fig. 3A and D). Transfection with miR-21 inhibitor increased cell apoptosis and decreased cell viability following combined treatment with Dox and LIUS (Fig. 3B and C), whereas miR-21 mimics increased cell viability and decreased apoptosis (Fig. 3E and F, respectively). The present results suggested that miR-21 regulated the effects of Dox and LIUS on apoptosis of Huh7 cells.

PTEN is a target of miR-21. Via bioinformatics prediction using TargetScan 7.0 and miRanda, a putative target site of miR-21 was identified in the 3'-UTR of PTEN mRNA, an important regulator of the AKT/mTOR pathway (Fig. 4A) (26) To investigate the interaction between miR-21 and the 3'-UTR of PTEN, a luciferase assay was performed. The WT or Mut 3'-UTR sequences of PTEN were cloned upstream of a luciferase gene and the constructed plasmids were transfected into Huh7 cells together with miR-21 inhibitor or mimics. The results of a dual-luciferase reporter assay suggested that miR-21 mimics suppressed the luciferase activity by $\sim 70 \%$ compared with control mimics. Conversely, miR-21 inhibitor increased the luciferase activity by $\sim 3$-fold (Fig. 4B). In contrast, transfection with miR-21 mimics or inhibitor did not affect the luciferase activity of a plasmid carrying the Mut 3'-UTR sequence of PTEN (Fig. 4B). In line with the luciferase assay results, western blotting suggested that miR-21 inhibitor enhanced the protein expression levels of PTEN, whereas miR-21 mimics decreased the protein expression levels of PTEN (Fig. 4C).

Treatment with LIUS increases sensitivity of cells to Dox via the ROS/miR-21/PTEN axis. PTEN is a tumor suppressor gene, and tumor growth is decreased following overexpression of PTEN $(27,28)$. To determine whether LIUS could enhance PTEN expression via the ROS/miR-21 pathway in HCC cells, Huh7 cells were treated with LIUS and/or Dox. After 48 h, western blot analysis was performed. Dox combined with LIUS increased the protein expression levels of PTEN, in line with the present results suggesting that LIUS suppressed cell viability and survival (Fig. 5A). Notably, NAC, a ROS inhibitor, 
A WT PTEN 3'-UTR: 3'...ACAACGUCUUCCAAGUAAGGACAUG-..-GgGUUA... 5' hsa-miR-21: 5' ...AGUUGUAG-...-.-.UCAG-..........-ACUAUUCGAU... 3'

Mut PTEN 3'-UTR: 3'...AGUUACGAUUCCAAGUAAGGACAUG--.-GGgUUA... 5'
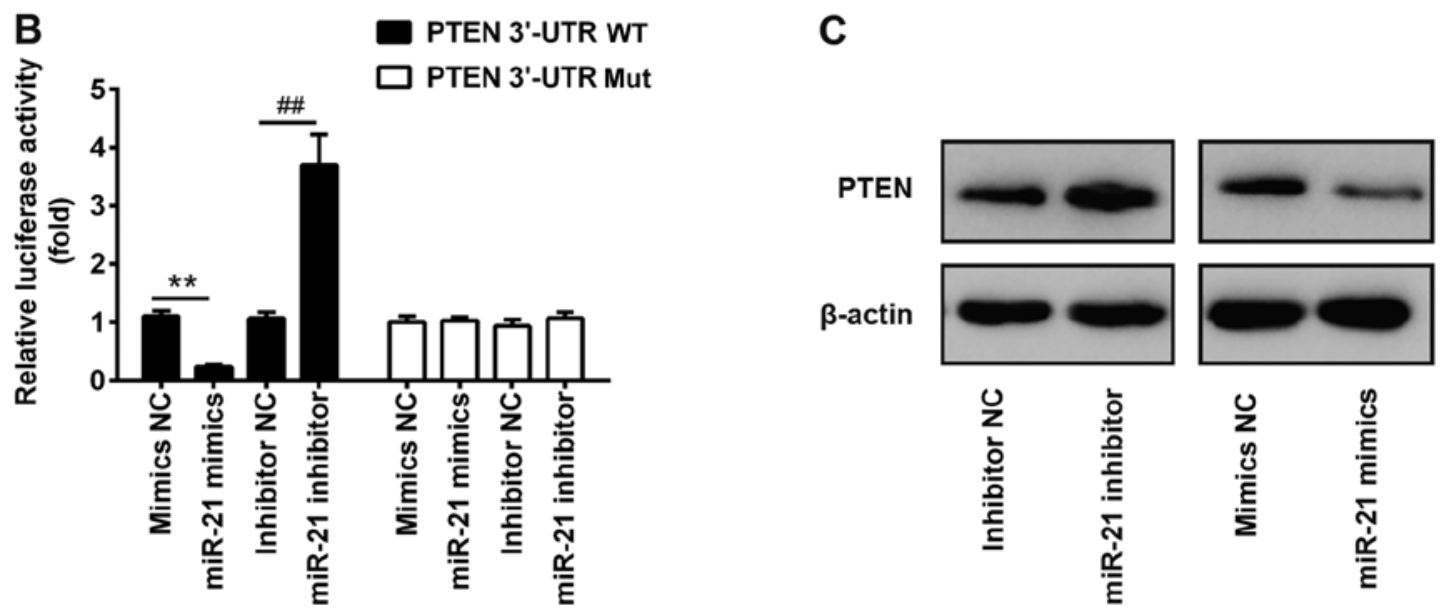

Figure 4. PTEN mRNA is a target of miR-21. (A) Schematic diagram of the 3'-UTR of PTEN as a putative target of miR-21. (B) Relative luciferase activity in Huh7 cells transfected with miR-21 inhibitor or miR-21 mimics and with firefly luciferase reporter plasmids containing the WT or Mut 3'-UTR of PTEN. ${ }^{* *} \mathrm{P}<0.01$ vs. mimics NC; ${ }^{\# \#} \mathrm{P}<0.01$ vs. inhibitor NC. (C) Huh7 cells were transfected with miR-21 inhibitor or miR-21 mimics, and the protein expression levels of PTEN were detected by western blotting. miR-21, microRNA-21; Mut, mutant; NC, negative control; PTEN, phosphatase and tensin homolog; UTR, untranslated region; WT, wild-type.

significantly decreased the protein expression levels of PTEN following combined treatment with Dox and LIUS (Fig. 5B). A previous study reported that miR-21 regulated the expression of PTEN and phosphorylation of its downstream kinase AKT, and that the reduction of $\mathrm{p}-\mathrm{AKT}$ was associated with enhanced chemosensitivity (29). To investigate the effects of Dox and LIUS cotreatment on activation of the AKT/mTOR pathway in Huh7, western blot analysis was performed. The present results suggested that the phosphorylation levels of AKT and mTOR were significantly decreased following combined treatment with Dox and LIUS compared with in the control group (Fig. 5C). However, treatment with NAC reversed this effect, suggesting that activation of the PTEN/AKT/mTOR pathway following treatment with Dox and LIUS was dependent on the accumulation of ROS. Collectively, the present results provided novel insights into the mechanism underlying the combination of LIUS and chemotherapy. Notably, LIUS was identified to promote chemotherapy sensitivity, inducing apoptosis of HCC cells and increasing the antitumor effects of Dox via the ROS/miR-21/PTEN pathway.

\section{Discussion}

In China, the incidence of HCC is increasing; in total, $\sim 466,000$ patients are diagnosed with HCC every year and it leads to $\sim 422,000$ cases of mortality (30). Surgery is an effective approach to treat HCC; however, it is suitable only for patients with early-stage HCC (31). In contrast, for patients with late-stage HCC, the available treatments are limited (32). Transarterial chemoembolization represents a standard treatment for patients with advanced HCC (33). However, patients with HCC treated with Dox or sorafenib exhibit resistance to chemotherapy (34). Epigenetic alterations, cellular export of drugs and evasion of apoptosis are frequently identified in resistant $\mathrm{HCC}$ cells, and these processes markedly limit the effectiveness of chemotherapy (35). Therefore, it is necessary to develop novel strategies to improve the effect of chemotherapy and prevent chemoresistance.

Ultrasound is a therapeutic approach that has been used in recent decades, and the identification of the optimal parameters is necessary for an effective treatment (36). Although LIUS has been demonstrated to be an effective anticancer treatment (12), high intensity focused ultrasound represents an additional non-invasive therapy to treat cancer (37). LIUS is characterized by a decreased intensity, and may alter the tumor environment and gene expression (38). However, the molecular mechanisms underlying ultrasound therapy remain unclear. Previous studies have demonstrated that the biological effects induced by ultrasound are primarily caused by thermal effects, inertial cavitation and ROS accumulation $(12,39)$. Thermal effects and inertial cavitation may cause protein denaturation and tissue damage $(39,40)$. The association between ultrasound treatment and ROS production has attracted increasing attention (41). A previous study on HCC revealed that LIUS increases ROS production, decreasing chemotherapy resistance and increasing the cellular uptake of DNA-damaging drugs (22). In line with these previous studies, the present results suggested that treatment with LIUS exhibited synergistic effects with Dox, and increased the sensitivity of HCC cells to Dox, promoting apoptosis of Huh7 cells.

ROS has been reported to regulate miRNAs involved in tumorigenesis; however, the association between ROS-induced miRNA dysregulation and chemotherapy resistance remains unclear. In the present study, ROS were identified to decrease the 
A

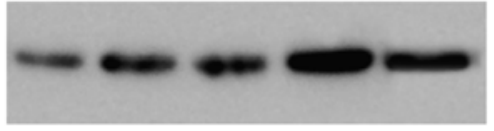

$\beta$-actin

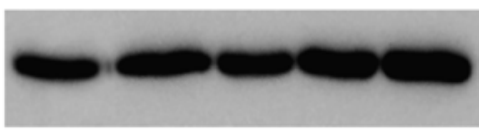

B

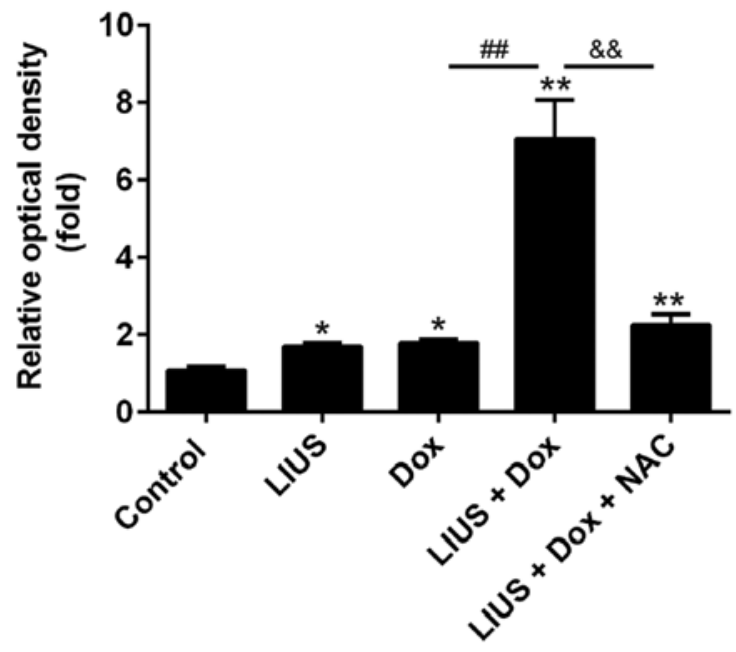

C
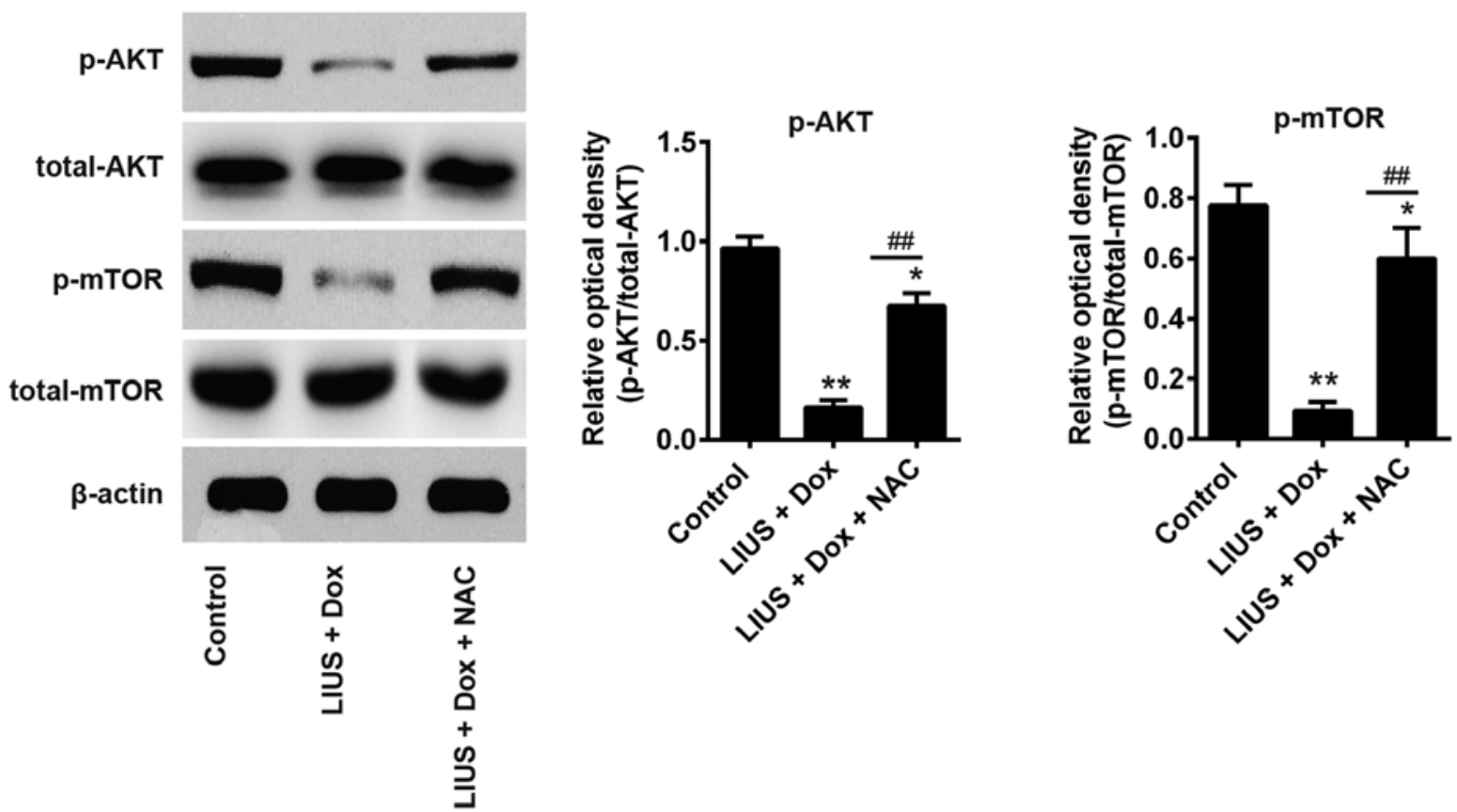

Figure 5. Treatment with LIUS enhances sensitivity to Dox via the reactive oxygen species/miR-21/PTEN axis. (A) Huh7 cells were treated with various compounds and the protein expression levels of PTEN were analyzed by western blotting. (B) Semi-quantification of the protein expression levels of PTEN normalized to $\beta$-actin. (C) Protein expression levels of p-AKT, AKT, p-mTOR and mTOR were measured by western blotting, and the protein expression levels were semi-quantified using ImageJ. ${ }^{*} \mathrm{P}<0.05,{ }^{* *} \mathrm{P}<0.01$ vs. control; ${ }^{\# \#} \mathrm{P}<0.01$ vs. Dox group; \&\& $\mathrm{P}<0.01$ vs. LIUS $+\mathrm{Dox}$ group. AKT, AKT serine/threonine kinase; Dox, doxorubicin; LIUS, low intensity ultrasound; miR-21, microRNA-21; mTOR, mechanistic target of rapamycin kinase; NAC, N-acetylcysteine; p-, phosphorylated; PTEN, phosphatase and tensin homolog; t-, total.

expression levels of miR-21 and treatment with NAC reversed this effect. miR-21 is an oncogene, and was identified to be upregulated in various types of cancer $(22,42)$. miR-21 regulates cancer cell proliferation, migration and various anti-apoptotic processes $(22,43,44)$. In the present study, the expression levels of miR-21 were significantly decreased following treatment with LIUS, as identified by microarray analysis. Furthermore, the present results suggested that the expression levels of PTEN were increased following miR-21 knockdown. PTEN is a tumor suppressor gene that has attracted increasing attention in cancer therapy (45). Additionally the PTEN/AKT signaling pathway has been identified to regulate cell growth and survival (45). In line with these previous studies, the present results suggested that treatment with LIUS increased the expression levels of PTEN by suppressing miR-21 expression and increased the sensitivity of HCC cells to Dox.

To the best of our knowledge, the present study is the first to suggest that LIUS combined with the chemotherapy drug Dox may induce apoptosis of HCC cells, increase chemotherapy sensitivity and exhibit potent antitumor effects. ROS production increased following treatment with LIUS and this decreased the expression levels of miR-21. The present results suggested that the expression levels of PTEN were regulated by the ROS/miR-21 axis, suggesting that LIUS affected tumor 
cell survival by regulating the PTEN/AKT signaling pathway. Collectively, this study provided novel insights into the molecular mechanism underlying the role of LIUS in promoting the effects of chemotherapy. In particular, treatment with LIUS increased chemotherapy sensitivity via the ROS/miR-21/PTEN pathway. The present results suggested that the combined treatment with LIUS and Dox may represent a novel strategy to treat HCC.

\section{Acknowledgements}

Not applicable.

\section{Funding}

The present study was supported by The Nature Fund Projects of The Inner Mongolia Autonomous Region (grant no. 2016MS08105).

\section{Availability of data and materials}

All data generated or analyzed during the present study are included in this published article.

\section{Authors' contributions}

CX, HZ and YZ performed the experiments, analyzed the data and wrote the paper. HZ designed the present study and provided experimental materials. All authors read and approved the final manuscript.

\section{Ethics approval and consent to participate}

Not applicable.

\section{Patient consent for publication}

Not applicable.

\section{Competing interests}

The authors declare that they have no competing interests.

\section{References}

1. Yii AC, Tan GL, Tan KL, Lapperre TS and Koh MS: Fixed airways obstruction among patients with severe asthma: Findings from the Singapore general hospital-severe asthma phenotype study. BMC Pulm Med 14: 191, 2014.

2. Lurje G, Lesurtel M and Clavien PA: Multimodal treatment strategies in patients undergoing surgery for hepatocellular carcinoma. Dig Dis 31: 112-117, 2013.

3. de Lope CR, Tremosini S, Forner A, Reig M and Bruix J: Management of HCC. J Hepatol 56 Suppl 1: S75-S87, 2012.

4. Giordano S and Columbano A: Met as a therapeutic target in HCC: Facts and hopes. J Hepatol 60: 442-452, 2014.

5. Liu L, Chen H, Wang M, Zhao Y, Cai G, Qi X and Han G: Combination therapy of sorafenib and TACE for unresectable HCC: A systematic review and meta-analysis. PLoS One 9: e91124, 2014.

6. Nishida N, Kitano M, Sakurai T and Kudo M: Molecular mechanism and prediction of sorafenib chemoresistance in human hepatocellular carcinoma. Dig Dis 33: 771-779, 2015.

7. Momparler RL, Karon M, Siegel SE and Avila F: Effect of adriamycin on DNA, RNA, and protein synthesis in cell-free systems and intact cells. Cancer Res 36: 2891-2895, 1976.
8. Rivankar S: An overview of doxorubicin formulations in cancer therapy. J Cancer Res Ther 10: 853-858, 2014.

9. Xu T, Zhang J, Chen W, Pan S, Zhi X, Wen L, Zhou Y, Chen BW, Qiu J, Zhang Y, et al: ARK5 promotes doxorubicin resistance in hepatocellular carcinoma via epithelial-mesenchymal transition. Cancer Lett 377: 140-148, 2016.

10. Pan JX, Wang F and Ye LY: Doxorubicin-induced epithelial-mesenchymal transition through SEMA 4A in hepatocellular carcinoma. Biochem Biophys Res Commun 479: 610-614, 2016.

11. Zhou Y, Liang C, Xue F, Chen W, Zhi X, Feng X, Bai X and Liang T: Salinomycin decreases doxorubicin resistance in hepatocellular carcinoma cells by inhibiting the $\beta$-catenin/TCF complex association via FOXO3a activation. Oncotarget 6 : 10350-10365, 2015.

12. Wood AK and Sehgal CM: A review of low-intensity ultrasound for cancer therapy. Ultrasound Med Biol 41: 905-928, 2015.

13. Lv Y, Fang M, Zheng J, Yang B, Li H, Xiuzigao Z, Song W, Chen Y and Cao W: Low-intensity ultrasound combined with 5-aminolevulinic acid administration in the treatment of human tongue squamous carcinoma. Cell Physiol Biochem 30: 321-333, 2012.

14. Fan H, Li H, Liu G, Cong W, Zhao H, Cao W and Zheng J: Doxorubicin combined with low intensity ultrasound suppresses the growth of oral squamous cell carcinoma in culture and in xenografts. J Exp Clin Cancer Res 36: 163, 2017.

15. Lan J, Huang Z, Han J, Shao J and Huang C: Redox regulation of microRNAs in cancer. Cancer Lett 418: 250-259, 2018.

16. Xiao Y, Yan W, Lu L, Wang Y, Lu W, Cao Y and Cai W: p38/p53/miR-200a-3p feedback loop promotes oxidative stress-mediated liver cell death. Cell Cycle 14: 1548-1558, 2015.

17. Yang $\mathrm{H}$, Li TW, Zhou Y, Peng H, Liu T, Zandi E, Martínez-Chantar ML, Mato JM and Lu SC: Activation of a novel c-Myc-miR27-prohibitin 1 circuitry in cholestatic liver injury inhibits glutathione synthesis in mice. Antioxid Redox Signal 22: 259-274, 2015.

18. Jin F, Wang Y, Li M, Zhu Y, Liang H, Wang C, Wang F, Zhang CY, Zen K and Li L: MiR-26 enhances chemosensitivity and promotes apoptosis of hepatocellular carcinoma cells through inhibiting autophagy. Cell Death Dis 8: e2540, 2017.

19. Zhang C, Liu X, Qiang H, Li K, Wang J, Chen D and Zhuang Y: Inhibitory effects of rosa roxburghii tratt juice on in vitro oxidative modification of low density lipoprotein and on the macrophage growth and cellular cholesteryl ester accumulation induced by oxidized low density lipoprotein. Clin Chim Acta 313: 37-43, 2001.

20. Livak KJ and Schmittgen TD: Analysis of relative gene expression data using real-time quantitative PCR and the 2(-Delta Delta C(T)) method. Methods 25: 402-408, 2001

21. Hu Z, Lv G, Li Y, Li E, Li H, Zhou Q, Yang B and Cao W: Enhancement of anti-tumor effects of 5-fluorouracil on hepatocellular carcinoma by low-intensity ultrasound. J Exp Clin Cancer Res 35: 71, 2016.

22. Pfeffer SR, Yang $\mathrm{CH}$ and Pfeffer LM: The role of miR-21 in cancer. Drug Dev Res 76: 270-277, 2015.

23. He C, Dong X, Zhai B, Jiang X, Dong D, Li B, Jiang H, Xu S and Sun X: MiR-21 mediates sorafenib resistance of hepatocellular carcinoma cells by inhibiting autophagy via the PTEN/Akt pathway. Oncotarget 6: 28867-28881, 2015 .

24. Li ZB, Li ZZ, Li L, Chu HT and Jia M: MiR-21 and miR-183 can simultaneously target SOCS6 and modulate growth and invasion of hepatocellular carcinoma (HCC) cells. Eur Rev Med Pharmacol Sci 19: 3208-3217, 2015.

25. Huang CS, Yu W, Cui H, Wang YJ, Zhang L, Han F and Huang T: Increased expression of miR-21 predicts poor prognosis in patients with hepatocellular carcinoma. Int J Clin Exp Pathol 8: 7234-7238, 2015.

26. Lim HJ, Crowe $P$ and Yang JL: Current clinical regulation of $\mathrm{PI} 3 \mathrm{~K} / \mathrm{PTEN} / \mathrm{Akt} / \mathrm{mTOR}$ signalling in treatment of human cancer. J Cancer Res Clin Oncol 141: 671-689, 2015.

27. Leslie NR and Downes CP: PTEN function: How normal cells control it and tumour cells lose it. Biochem J 382: 1-11, 2004.

28. Yu HG, Ai YW, Yu LL, Zhou XD, Liu J, Li JH, Xu XM, Liu S, Chen J, Liu F, et al: Phosphoinositide 3-kinase/Akt pathway plays an important role in chemoresistance of gastric cancer cells against etoposide and doxorubicin induced cell death. Int J Cancer 122: 433-443, 2008.

29. Yang SM, Huang C, Li XF, Yu MZ, He Y and Li J: miR-21 confers cisplatin resistance in gastric cancer cells by regulating PTEN. Toxicology 306: 162-168, 2013. 
30. Chen W, Zheng R, Baade PD, Zhang S, Zeng H, Bray F, Jemal A, Yu XQ and He J: Cancer statistics in China, 2015. CA Cancer J Clin 66: 115-132, 2016.

31. Kirstein MM and Vogel A: The pathogenesis of hepatocellular carcinoma. Dig Dis 32: 545-553, 2014.

32. Rich NE, Yopp AC and Singal AG: Medical management of hepatocellular carcinoma. J Oncol Pract 13: 356-364, 2017.

33. Reig M, Darnell A, Forner A, Rimola J, Ayuso C and Bruix J: Systemic therapy for hepatocellular carcinoma: The issue of treatment stage migration and registration of progression using the BCLC-refined RECIST. Semin Liver Dis 34: 444-455, 2014.

34. Pan ST, Li ZL, He ZX, Qiu JX and Zhou SF: Molecular mechanisms for tumour resistance to chemotherapy. Clin Exp Pharmacol Physiol 43: 723-737, 2016.

35. Lohitesh K, Chowdhury R and Mukherjee S: Resistance a major hindrance to chemotherapy in hepatocellular carcinoma: An insight. Cancer Cell Int 18: 44, 2018.

36. Draper DO: Facts and misfits in ultrasound therapy: Steps to improve your treatment outcomes. Eur J Phys Rehabil Med 50: 209-216, 2014

37. Wu F: High intensity focused ultrasound: A noninvasive therapy for locally advanced pancreatic cancer. World J Gastroenterol 20: 16480-16488, 2014

38. Xia B, Zou Y, Xu Z and Lv Y: Gene expression profiling analysis of the effects of low-intensity pulsed ultrasound on induced pluripotent stem cell-derived neural crest stem cells. Biotechnol Appl Biochem 64: 927-937, 2017.
39. Duco W, Grosso V, Zaccari D and Soltermann AT: Generation of ROS mediated by mechanical waves (ultrasound) and its possible applications. Methods 109: 141-148, 2016.

40. Jang HJ, Lee JY, Lee DH, Kim WH and Hwang JH: Current and future clinical applications of high-intensity focused ultrasound (HIFU) for pancreatic cancer. Gut Liver 4 Suppl 1: S57-S61, 2010.

41. Shi J, Chen Z, Wang B, Wang L, Lu T and Zhang Z: Reactive oxygen species-manipulated drug release from a smart envelope-type mesoporous titanium nanovehicle for tumor sonodynamic-chemotherapy. ACS Appl Mater Interfaces 7: 28554-28565, 2015.

42. Ma X, Conklin DJ, Li F, Dai Z, Hua X, Li Y, Xu-Monette ZY, Young KH, Xiong W, Wysoczynski M, et al: The oncogenic microRNA miR-21 promotes regulated necrosis in mice. Nat Commun 6: 7151, 2015 .

43. Melnik BC: MiR-21: An environmental driver of malignant melanoma? J Transl Med 13: 202, 2015.

44. Sekar D, Krishnan R, Thirugnanasambantham K, Rajasekaran B, Islam VI and Sekar P: Significance of microRNA 21 in gastric cancer. Clin Res Hepatol Gastroenterol 40: 538-545, 2016.

45. Worby CA and Dixon JE: Pten. Annu Rev Biochem 83: 641-669, 2014.

This work is licensed under a Creative Commons Attribution-NonCommercial-NoDerivatives 4.0 International (CC BY-NC-ND 4.0) License. 